\title{
Homozygosity analysis in subjects with autistic spectrum disorder
}

\author{
AHMAD ADI $^{1}$, BASMA TAWIL $^{1}$, MOHAMMED ALDOSARI $^{2}$, JAMEELA SHINWARI $^{1}$, \\ MICHAEL NESTER $^{2}$, HISHAM ALDHALAAN ${ }^{2,3}$, HUSSAIN ALSHAMRANI ${ }^{2,4}$, \\ MANAR GHANNAM ${ }^{2}$, BRIAN MEYER ${ }^{5}$ and NADA AL TASSAN ${ }^{1}$ \\ ${ }^{1}$ Behavioral Genetics Unit, Department of Genetics, King Faisal Specialist Hospital and Research Center; \\ ${ }^{2}$ Center for Autism Research, King Faisal Specialist Hospital; Departments of ${ }^{3}$ Neuroscience, ${ }^{4}$ Pediatrics, and ${ }^{5}$ Genetics, \\ King Faisal Specialist Hospital and Research Center, Riyadh 11211, Saudi Arabia
}

Received August 26, 2014; Accepted March 23, 2015

DOI: $10.3892 / \mathrm{mmr} .2015 .3663$

\begin{abstract}
Autistic spectrum disorder (ASD) is a complex neurodevelopmental disorder that results in social and communication impairments, as well as repetitive and stereotyped patterns. Genetically, ASD has been described as a multifactorial genetic disorder. The aim of the present study was to investigate possible susceptibility loci of ASD, utilizing the highly consanguineous and inbred nature of numerous families within the population of Saudi Arabia. A total of 13 multiplex families and 27 affected individuals were recruited and analyzed using Affymetrix GeneChip ${ }^{\circledR}$ Mapping $250 \mathrm{~K}$ and 6.0 arrays as well as Axiom arrays. Numerous regions of homozygosity were identified, including regions in genes associated with synaptic function and neurotransmitters, as well as energy and mitochondria-associated genes, and developmentally-associated genes. The loci identified in the present study represent regions that may be further investigated, which could reveal novel changes and variations associated with ASD, reinforcing the complex inheritance of the disease.
\end{abstract}

\section{Introduction}

Autistic spectrum disorder (ASD) (MIM 209850) is a complex neurodevelopmental disorder that results in social and communication impairment, as well as repetitive and stereotyped patterns $(1,2)$. Other non-psychiatric symptoms of ASD include intellectual disability, motor delay, hypotonia and seizures (2). Individuals affected with ASD have also been reported to exhibit neuroanatomical structure alterations (3). Epidemiologically, the frequency of ASD is estimated to be $\sim 60$ cases per 10,000 children, increasing from early epide-

Correspondence to: Dr Nada Al Tassan, Behavioral Genetics Unit, Department of Genetics, King Faisal Specialist Hospital and Research Center, P.O. Box 3354, Riyadh 11211, Saudi Arabia E-mail: naltassan@kfshrc.edu.sa

Key words: autistic spectrum disorder, homozygosity, loci, genes miological studies that originally reported $2-5$ cases per 10,000 children worldwide $(2,4)$.

Genetically, ASD has been described as a multifactorial genetic disorder, which in certain cases is associated with a specific mutation or syndrome, such as fragile $\mathrm{X}$ syndrome (Xq27.3) (5), tuberous sclerosis (9q or 16p) (6) and Angelman/Prader-Willi syndrome (15q11-q13 chromosomal abnormality) $(7,8)$. Mutations in genes that encode proteins associated with synaptic transmission and activity, including NLGN3/NLGN4 and SHANK3, have been reported in patients with ASD (2,9-11). Other reported changes include protein synthesis-associated genes (FMR1 and PTEN), transcription factors $(M E F 2)$ (11), and neurotransmitter proteins and receptors [glutamate and gamma-aminobutyric acid (GABA) receptors] (10). Previous reports have linked ASD to dysfunctional energy metabolism in the central nervous system (CNS), which may describe changes in mitochondrial DNA, or nuclear DNA associated with mitochondrial function (10). In addition, there are numerous cases where ASD appears to arise from a de novo genetic alteration (9).

In the effort to characterize novel genetic alterations associated with ASD, the aim of the present study was to investigate potential susceptibility loci of ASD, utilizing the highly consanguineous and inbred nature of numerous families within the population of Saudi Arabia. Families with ASD-affected individuals were recruited in the present study, and loss of heterozygosity analyses were performed. The present study presents possible regions that may be linked to ASD, as obtained from a loss of heterozygosity analysis.

\section{Materials and methods}

Patients and samples. The present study was performed under the approval of the tertiary care center King Faisal Specialist Hospital and Research Center (KFSHRC RAC no. 2080001; Riyadh, Saudi Arabia). The present study was a collaboration between the departments of Genetics and Neuroscience, and the Autism Research Center at KFSHRC. Families with $>1$ affected individual, regarded as multiplex families, were identified and recruited. The majority of the families recruited contained consanguineous marriages. Examples of family pedigrees from a selection of the recruited families are 


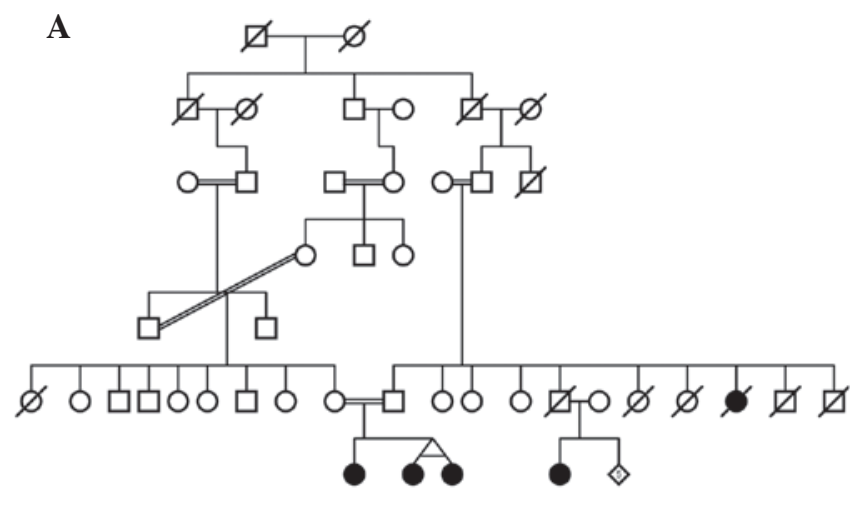

B

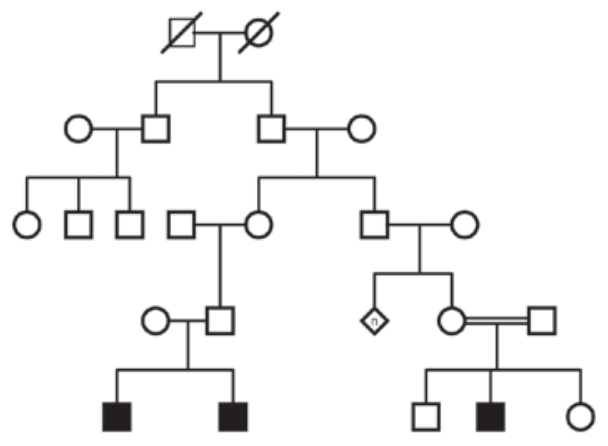

C

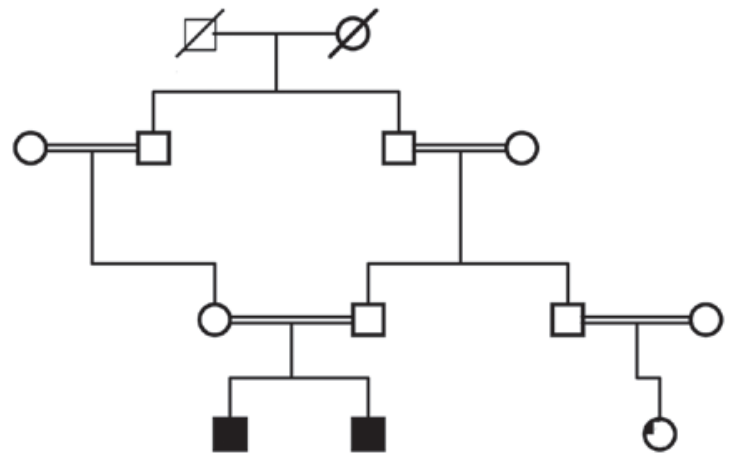

Figure 1. Examples of family pedigrees. Consanguinity is evident in these families and the autosomal recessive pattern is observed from pedigrees ( $\sim 60 \%$ of cases are consanguineous). (A) Pedigree presents 3 affected female siblings including twins. (B) Pedigree where the parents of one affected individual are related. There are 2 affected males diagnosed with ASD. (C) Pedigree with two male siblings diagnosed with ASD. Double lines indicate consanguinity. ASD, autistic spectrum disorder.

shown in Fig. 1. Diagnosis of ASD was made at KFSHRC, according to the ADI-R and DSM-VI criteria $(1,2)$. Families with individuals diagnosed with ASD secondary to known genetic or metabolic disorders were excluded. Blood samples (3-5 ml) were obtained in EDTA tubes from family members for subsequent genetic analysis. The family members provided written informed consent in adherence to institutional and international guidelines (RAC\#2080001).

DNA extraction. DNA was extracted from the peripheral blood samples using the Gentra Systems Puregene DNA Isolation kit (Qiagen, Valencia, CA, USA), according to the manufacturer's instructions. DNA concentration was determined spectrophotometrically using a Nanodrop (Thermo Fisher Scientific, Waltham, MA, USA).

Loss of heterozygosity analysis. All DNA samples were genotyped using GeneChip ${ }^{\circledR}$ Mapping 250K and 6.0 arrays, and the Axiom Genome-Wide CEU 1 Human Mapping Array (Affymetrix, Santa Clara, CA, USA), according to the manufacturer's instructions. Briefly, 25-250 ng of high-quality genomic DNA was digested using the restriction endonuclease StyI for the $250 \mathrm{~K}$ array, and StyI and NspI restriction endonucleases for the 6.0 array. The products were then ligated with an adaptor. Generic primers that recognize the adaptor sequence were used to amplify the adaptor-ligated DNA fragments in the recommended size range. The amplified polymerase chain reaction products were then fragmented with the Affymetrix fragmentation reagent to 50-200 bp, and end-labeled with biotinylated dideoxyadenosine triphosphate using terminal deoxynucleotidyl transferase. The end-labeled fragments were then hybridized to
GeneChip ${ }^{\circledR}$ Human Mapping 250K or 6.0 arrays. For the Axiom Set, the target DNA was amplified using the Axiom 2.0 Amp kit and then fragmented according to the manufacturer's recommendations (Affymetrix). The pellets were then re-suspended and hybridized, and genotyping was performed using the GeneTitan ${ }^{\circledR}$ Multi-Channel Instrument (Affymetrix).

GeneChip ${ }^{\circledR}$ Operating software (version 1.4) and Genotyping Console ${ }^{\mathrm{TM}}$ (GTC; version 3.01) software (Affymetrix) were used for primary data analysis, normalization against internal control features on the chip, genotype calling and quality control (overall single nucleotide polymorphism call rate, 95-99\%). Data generated from the arrays were then analyzed using Affymetrix Genotyping Console (version 3.01) and/or a homozygosity mapper program (http:// www.homozygositymapper.org/) (12). Regions of shared homozygosity between affected individuals within a family, but not shared with unaffected individuals, were identified. Also, regions of shared homozygosity between affected individuals from different families were identified. The cutoff characteristic was that the region had to be shared between all of the affected individuals from at least four different families. Unaffected individuals from three random families were used as controls. Regions $<2 \mathrm{Mb}$ were excluded from the analysis, and gender-specific chromosomes were not analyzed.

Identified regions of homozygosity were entered into the Suspects Candidate Gene Search v.28.3 (http://www. cgem.ed.ac.uk/resources/suspects/) from the University of Edinburgh (Edinburgh, UK), in order to prioritize genes in an order of their reported relevance to the development of ASD (12). The top three candidate genes were reported in the present study. 
Table I. Regions of homozygosity identified in the analysis of multiplex families $(n=13)$.

\begin{tabular}{|c|c|c|c|}
\hline Chromosome & Coordinates & Top 3 genes & Family ID \\
\hline \multirow[t]{4}{*}{1} & $17,606,235-20,525,209$ & TAS1R2, HTR6, PLA2G2F & F14 \\
\hline & $66,592,949-70,541,226$ & NP_065999,NP_060238,SLC35D1 & $\mathrm{F} 14$ \\
\hline & $90,600,674-10,477,047$ & ATP6VOB, SLC1A7, ATPIF 1 & $\mathrm{~F} 20$ \\
\hline & $208,362,435-211,304,878$ & NP_061134,ATF3, RPS6KC1 & F8 \\
\hline \multirow[t]{5}{*}{2} & $16,280,027-18,724,599$ & KCNS3, SMC6L1, VSNL1 & F14 \\
\hline & $111,883,724-114,024,469$ & SLC20A1, MERTK, NP_116213 & $\mathrm{F} 23$ \\
\hline & $162,797,863-165,675,258$ & KCNH7, FAP, NP_775783 & F23 \\
\hline & $129,417,474-133,043,938$ & NP_079305,PLEKHB2, CFC1 & F6 \\
\hline & $180,338,192-192,340,990$ & ITGAV, NAB1, COL3A1 & F7 \\
\hline \multirow[t]{2}{*}{3} & $49,088,836-51,462,363$ & $B S N, D O C K 3, L A M B 2$ & $\mathrm{~F} 20$ \\
\hline & $155,790,787-160,134,049$ & Q8NGV9, SSR3, GMPS & F14 \\
\hline \multirow[t]{5}{*}{4} & $3,691,191-5,842,856$ & $M S X 1, C Y T L 1, S T K 32 B$ & F14 \\
\hline & $37,930,813-44,149,564$ & CHRNA9, APBB2, SLC3OA9 & F14 \\
\hline & $75,534,780-81,987,898$ & FRAS1, ANTXR2, GK2 & F7 \\
\hline & $91,479,821-96,553,748$ & PDLIM5, GRID2, BMPRIB & $\mathrm{F} 3$ \\
\hline & $127,483,173-131,017,058$ & SLC25A31, PHF17, PDZK6 & F6 \\
\hline \multirow[t]{9}{*}{5} & $10,671,102-12,018,348$ & $C T N N D 2, D A P$ & F14 \\
\hline & $13,115,454-15,292,186$ & DNAH5, TRIO, ANKH & $\mathrm{F} 23$ \\
\hline & $43,721,462-46,644,204$ & HCN1, FGF10, MRPS30 & F14 \\
\hline & $63,181,011-65,623,491$ & TRIM23, ADAMTS6, HTRIA & F14-F7 \\
\hline & $70,580,012-78,664,650$ & $F 2 R L 1, F 2 R L 2, F 2 R$ & F14 \\
\hline & $91,871,294-95,831,186$ & KIAA0372, PCSK1, NR2F 1 & F14 \\
\hline & $105,398,456-108,334,980$ & EFNA5, FBXL17 & F6 \\
\hline & $110,309,108-113,085,059$ & SRP19, APC, WDR36 & $\mathrm{F} 23$ \\
\hline & $154,784,618-157,912,731$ & $A D A M 19, C R S P 9, S G C D$ & $\mathrm{~F} 23$ \\
\hline \multirow[t]{6}{*}{6} & $43,852,052-46,694,741$ & DSCR1L1, ENPP4, SLC35B2 & F20 \\
\hline & $51,472,664-58,554,555$ & PKHD1, MCM3, ICK & F6-F14 \\
\hline & $61,784,585-65,361,195$ & PTP4A1, KHDRBS2, GLULD1 & F14 \\
\hline & $89,467,426-91,642,040$ & GABRR2, GABRR1, MDN1 & F14 \\
\hline & $117,801,917-120,016,046$ & MAN1A1, DCBLD1, C6orf60 & F14 \\
\hline & $128,741,476-132,437,814$ & AKAP7, CTGF, LAMA2 & F14 \\
\hline \multirow[t]{9}{*}{7} & $44,167-3,935,550$ & GPR146, LFNG, CHST12 & $\mathrm{F} 23$ \\
\hline & $33,956,386-38,816,634$ & SEPT7, AMPH, GPR154 & F14 \\
\hline & $39,251,002-42,234,071$ & $G L I 3, I N H B A, C D C 2 L 5$ & F14 \\
\hline & $78,299,408-93,347,267$ & GRM3, SRI, CACNA2DI & F14 \\
\hline & $88,881,477-91,474,214$ & PFTK1, CYP51A1, CLDN12 & F14 \\
\hline & $118,385,860-122,485,086$ & SLC13A1, KCND2, WNT16 & $\mathrm{F} 1$ \\
\hline & $132,226,627-137,446,264$ & SLC13A4, PTN, NUP205 & $\mathrm{F} 7$ \\
\hline & $143,159,897-147,967,548$ & CNTNAP2, TPK1, O60393 & F14 \\
\hline & $149,811,568-153,488,778$ & MLL3, ACCN3, KCNH2 & F14 \\
\hline \multirow[t]{4}{*}{8} & $95,013,089-99,313,599$ & UQCRB, GDF6, NP_057218 & F14 \\
\hline & $113,038,464-118,887,549$ & CSMD3, THRAP6, NP_115710 & F14 \\
\hline & $8,966,544-10,388,436$ & MSRA, TNKS, PPPIR3B & $\mathrm{F} 20$ \\
\hline & $29,493,059-31,510,288$ & GSR, WRN, LEPROTLI & $\mathrm{F} 23$ \\
\hline \multirow[t]{2}{*}{9} & $77,676,620-85,812,95$ & SLC28A3, NTRK2, TLE1 & F14 \\
\hline & $125,138,695-137,750,235$ & GRIN1, KCNT1, SLC25A25 & $\mathrm{F} 23$ \\
\hline \multirow[t]{4}{*}{10} & $24,259,097-26,357,328$ & GPR158, THNSL1, PRTFDC1 & F6 \\
\hline & $34,935,425-36,373,753$ & CREM,NP_699199,CUL2 & $\mathrm{F} 23$ \\
\hline & $56,919,560-59,481,992$ & ZWINT, ZWINTAS & F7 \\
\hline & $79,202,450-85,447,212$ & SFTPD, ANXA11, PPIF & $\mathrm{F} 23$ \\
\hline 11 & $43,773,141-51,682,438$ & RAPSN, PSMC3, SPII & F3 \\
\hline
\end{tabular}


Table I. Continued.

\begin{tabular}{|c|c|c|c|}
\hline Chromosome & Coordinates & Top 3 genes & Family ID \\
\hline 11 & $110,025,057-112,216,763$ & PTS, TIMM8B, DLAT & F23 \\
\hline 12 & $\begin{array}{l}4,042,363-10,283,711 \\
37,345,650-39,627,393\end{array}$ & $\begin{array}{l}A 2 M, C H D 4, T N F R S F 1 A \\
K I F 21 A, S L C 2 A 13, A B C D 2\end{array}$ & $\begin{array}{l}\text { F7 } \\
\text { F23 }\end{array}$ \\
\hline 15 & $\begin{array}{l}80,676,184-82,887,137 \\
95,798,343-101,692,828\end{array}$ & $\begin{array}{l}\text { AP } 3 B 2, \text { BTBD1, RPS17 } \\
\text { ADAMTS17, CHSY1, IGF1R }\end{array}$ & $\begin{array}{l}\text { F23 } \\
\text { F17 }\end{array}$ \\
\hline 16 & $\begin{array}{l}65,000,489-70,848,261 \\
6,392,511-8,255,216 \\
63,133,419-77,264,064\end{array}$ & $\begin{array}{l}\text { SLC12A4, CTCF, ATP6VOD1 } \\
\text { A2BP_HUMAN, Q8WZ91, Q14229 } \\
\text { SLC12A4, CTCF, ATP6VOD1 }\end{array}$ & $\begin{array}{l}\text { F1-F14 } \\
\text { F14 } \\
\text { F14 }\end{array}$ \\
\hline 17 & $\begin{array}{l}32,587,614-35,196,133 \\
21,890,916-27,461,374 \\
76,007,579-78,771,650\end{array}$ & $\begin{array}{l}\text { GPR158L1, ERBB2, NEUROD2 } \\
\text { SLC6A4, SLC13A2, UNC119 } \\
\text { SLC25A10, FOXK2, SLC16A3 }\end{array}$ & $\begin{array}{l}\text { F3 } \\
\text { F14 } \\
\text { F23 }\end{array}$ \\
\hline 18 & $36,589,650-38,808,690$ & $P I K 3 C 3$ & F23 \\
\hline 19 & $4,463,233-7,013,199$ & SLC25A23,NP_775908, PTPRS & F8 \\
\hline 20 & $13,821,260-16,084,725$ & FLRT3, C20orf133, Q9NQI9 & F14 \\
\hline 21 & $23,309,707-39,155,954$ & ATP5J, ATP5O, GRIK1 & F23 \\
\hline
\end{tabular}

Samples were genotyped using GeneChip ${ }^{\circledR}$ Mapping 250K and 6.0 arrays and the Axiom Set. Samples from affected and unaffected individuals from all 13 families were genotyped using GeneChip® Mapping 250K, 6.0 arrays and Axiom. ( 54 individuals, 27 affected subjects).

Table II. Regions of shared homozygosity obtained from analyzing all affected individuals from multiplex families using GeneChip ${ }^{\circledR}$ Mapping 250k and 6.0 arrays.

\begin{tabular}{cll}
\hline Chromosome & \multicolumn{1}{c}{ Coordinates and Axiom } & \multicolumn{1}{c}{ Top 3 genes } \\
\hline 1 & $102,688,721-105,073,165$ & COL11A1, AMY1A, AMY1A \\
2 & $134,545,725-136,584,137$ & ACMSD, LCT, MGAT5 \\
3 & $49,856,601-51,968,474$ & GRM2, DOCK3, SLC38A3 \\
7 & $77,339,194-80,531,718$ & SEMA3C, CD36, GNAI1 \\
11 & $47,240,293-49,279,489$ & PSMC3, RAPSN, SPI1 \\
& $48,549,401-50,779,621$ & FOLH1, TYRL, OR4C13 \\
15 & $25,581,119-27,659,946$ & APBA2, HERC2, OCA2 \\
\hline
\end{tabular}

\section{Results}

A total of 13 multiplex families were recruited and analyzed using GeneChip ${ }^{\circledR}$ Mapping $250 \mathrm{~K}$ and 6.0 arrays, and the Axiom Set. There were $>27$ affected individuals within the recruited families. A selection of the pedigrees is presented in Fig. 1.

Regions of homozygosity identified from the independent analysis of each family were spread across all chromosomes. The chromosome with the most regions of homozygosity was identified as chromosome 5 (Table I). Shared regions of homozygosity between the affected individuals from all of the families were also determined. There were seven regions that were shared between all individuals, with two regions on chromosome 11 (Table II). The number of genes in the regions identified ranged between 3 genes to several hundreds. Notably, no regions of homozygosity (above $2 \mathrm{Mb}$ in size) were observed on chromosomes 13, 14 and 22 as per the analysis criteria used.

\section{Discussion}

The regions of homozygosity identified in the present study contained numerous genes that have previously been reported as possible causes of ASD (13). The results included the following genes: GRIN1, GRIK1, GRID2, GPR158L1, GLULD1 and GRM3, which are associated with glutamate receptor and proteins, RAPSN, which is associated with acetylcholine receptors and transmission, and GABRRI and GABRR2, which are associated with GABA receptors (14). The results of the present study support the numerous reports that suggest ASD may be a synaptopathic disease (15). Furthermore, numerous genes in these regions are associated with mitochondria and are energy-associated, including ATP5J, SLC25A25, SLC25A31 and MRPS30, which have also been implicated in previous studies $(16,17)$. A third group of genes identified in these regions were consistent with embry- 
onic/development-associated genes, including, WNT 16 and NEUROD2, suggesting a possible early insult to the central nervous system in the developing embryo (18).

A number of the regions reported to be associated with the development of ASD include: i) Duplication or deletion of 16p11.2, which has been implicated in patients with ASD as well as in animal models, which may cause alterations in brain anatomy and behavior $(19,20)$; ii) a $1.5-\mathrm{Mb}$ microdeletion in region 14q23.2-23.3, which is associated with ASD, as well as spherocytosis (21); iii) a deletion at 2p15 (22); iv) an alteration at 8 p23.2 (23); and v) a 535-Kb deletion at 3p26.3 (24). However, the results of the present study did not indicate any alterations to these regions, suggesting that ASD-associated gene alterations differ across various populations.

As shown in Table II, the number of regions identified when analyzing all of the affected individuals from the different families was lower, as compared with the regions obtained from analyzing the affected individuals within their respective families. In addition, there were no regions that were universally present in all of the affected individuals from the different families. However, 7 shared regions were observed between certain affected individuals. This difference may be attributed to the fact that ASD is a genetically complex and multifactorial disorder, which concurs with numerous previous studies and reports regarding the genetics of ASD $(3,4,9)$. There may be more than one genetic change per family, and different genetic changes between families. Biallelic mutations have been reported in other disorders with complex phenotypes (25-27).

One of the challenges in studying the genetics of ASD in Saudi Arabia is that the Saudi population, as evident in the families that were recruited in the present study, is highly consanguineous and inbred. This represents a challenge in identifying genetic regions that are associated with ASD, as opposed to regions that may be normally shared as a result of identity by descent. In order to overcome this challenge, it has been proposed for future studies that appropriate tests and statistics are conducted prior to genetic analysis that allow for discrimination of these two types of regions, such as the coefficient of inbreeding (28).

Another challenge facing the genetic study of ASD is that ASD represents a variable spectrum, in which the milder forms may not be detected with conventional clinical methods. This is relevant to the results of the present study in that when the results of affected individuals are compared with normal controls, there may be missing/excluded regions. This may possibly be due to the controls having a mild form of ASD that the clinic was unable to detect. Further study of ASD genetics is therefore dependent on the development of more specific clinical tools. Another way to overcome this issue in future studies is to include information on clinically normal individuals, including their intelligence quotient, social interaction status and their school performance. As has been reported previously, the possibility of the presence of multiple phenotypes within the same family suggests involvement of multiple interacting loci, which adds to the complexity of the inheritance of ASD (28).

As mentioned previously, all of the regions identified in the present study were $>2 \mathrm{Mb}$. It is possible that more regions and possible genes may be identified for analysis by examining smaller regions. This is the aim for future studies by our group, as well as the inclusion of gender-associated chromosomes in the analyses.

Candidate autism genes usually have distinct biological roles and interactions. In order to attain an improved understanding regarding the pathogenesis of ASD, it is important to discover the location and functional role of autism-associated proteins in biological pathways, and hence in neuronal function and resulting behavior (29). An ASD interactome was generated to meet the obstacles caused by this hereditary neurological disorder, and to identify proteins that interact with ASD-associated proteins (30). Of note, when the ASD interactome was created, a high connectivity was identified between two ASD-associated proteins that initially appeared functionally unrelated, SHANK3 and TSC1. SHANK3 is an adapter protein in the post-synaptic regions that may have a role in the organization of the dendritic spine and synaptic junction, and $\mathrm{TSC} 1$ is a tuberous sclerosis 1 protein that regulates mechanistic (serine/threonine kinase) target of rapamycin, a promoter of protein synthesis. However, these two proteins share $\geq 21$ protein associates and were found to interact in a complex scaffold at the post-synaptic region (30), thus suggesting that various ASD-associated proteins may share a common key pathway associated with disease development. Similarly, the genes identified in the present study involve various categories of proteins that may also share a common molecular pathway, and may be further investigated through a protein-protein interaction assay. Furthermore, since ASD genes differ between populations, creating an interactome for these Saudi ASD proteins would be beneficial not only in the development of treatment, but also in developing powerful therapies for a specific ethnic group.

\section{Acknowledgements}

The present study was supported by the King Faisal Specialist Hospital and Research Center (KFSHRC) (RAC\# 2080001) and the Center for Autism Research. The authors of the present study would also like to acknowledge the genotyping core facility at KFSHRC.

\section{References}

1. American Psychiatric Association: Diagnostic and statistical manual of mental disorders (4th ed., text rev.), 2000.

2. Levy SE, Mandell DS and Schultz RT: Autism. Lancet 374: 1627-1638, 2009.

3. Bailey A, Le Couteur A, Gottesman I, et al: Autism as a strongly genetic disorder: evidence from a British twin study. Psychol Med 25: 63-77, 1995.

4. Bryson S: Epidemiology of autism: overview and issues outstanding. In: Handbook of Autism and Pervasive Developmental Disorders. 2nd edition. Cohen D and Volkmar F (eds). John Wiley and Sons, New York, pp41-46, 1997.

5. Rogers SJ, Wehner DE and Hagerman R: The behavioral phenotype in fragile $\mathrm{X}$ : symptoms of autism in very young children with fragile $X$ syndrome, idiopathic autism, and other developmental disorders. J Dev Behav Pediatr 22: 409-417, 2001.

6. Baker P, Piven J and Sato Y: Autism and tuberous sclerosis complex: prevalence and clinical features. J Autism Dev Disord 28: 279-285, 1998

7. Steffenburg S, Gillberg CL, Steffenburg U and Kyllerman M: Autism in Angelman syndrome: a population-based study. Pediatr Neurol 14: 131-136, 1996. 
8. Sutcliffe JS, Nurmi EL and Lombroso PJ: Genetics of childhood disorders: XLVII. Autism, part 6: duplication and inherited susceptibility of chromosome 15q11-q13 genes in autism. J Am Acad Child Adolesc Psychiatry 42: 253-256, 2003.

9. Geschwind DH: Autism: many genes, common pathways? Cell 135: 391-395, 2008.

10. Smith M, Spence MA and Flodman P: Nuclear and mitochondrial genome defects in autisms. Ann N Y Acad Sci 1151: 102-132, 2009.

11. Walsh CA, Morrow EM and Rubenstein JL: Autism and brain development. Cell 135: 396-400, 2008.

12. Adie EA, Adams RR, Evans KL, Porteous DJ and Pickard BS: Speeding disease gene discovery by sequence based candidate prioritization. BMC Bioinformatics 6: 55, 2005.

13. Freitag CM: The genetics of autistic disorders and its clinical relevance: A review of the literature. Mol Psychiatry 12: 2-22, 2007.

14. Tarabeux J, Kebir O, Gauthier J, et al; S2D team: Rare mutations in N-methyl-D-aspartate glutamate receptors in autism spectrum disorders and schizophrenia. Transl Psychiatry 1: e55, 2011.

15. Bourgeron T: A synaptic trek to autism. Curr Opin Neurobiol 19: 231-234, 2009.

16. Anitha A, Nakamura K, Thanseem I, et al: Brain region-specific altered expression and association of mitochondria-related genes in autism. Mol Autism 3: 12, 2012.

17. Tang G, Gutierrez Rios P, Kuo SH, et al: Mitochondrial abnormalities in temporal lobe of autistic brain. Neurobiol Dis 54: 349-361, 2013

18. Olson JM, Asakura A, Snider L, Hawkes R, Strand A, Stoeck J, Hallahan A, Pritchard J and Tapscott SJ: NeuroD2 is necessary for development and survival of central nervous system neurons. Dev Biol 234: 174-187, 2001.

19. Horev G, Ellegood J, Lerch JP, et al: Dosage-dependent phenotypes in models of $16 \mathrm{p} 11.2$ lesions found in autism. Proc Natl Acad Sci USA 108: 17076-17081, 2011.
20. Kumar RA, Marshall CR, Badner JA, et al: Association and mutation analyses of 16 p11.2 autism candidate genes. PLoS One 4: e4582, 2009.

21. Griswold AJ, Ma D, Sacharow SJ, et al: A de novo $1.5 \mathrm{Mb}$ microdeletion on chromosome 14q23.2-23.3 in a patient with autism and spherocytosis. Autism Res 4: 221-227, 2011.

22. Liu X, Malenfant P, Reesor C, et al: 2p15-p16.1 microdeletion syndrome: molecular characterization and association of the OTX1 and XPO1 genes with autism spectrum disorders. Eur J Hum Genet 19: 1264-1270, 2011.

23. Nucaro A, Pisano T, Chillotti I, Montaldo C and Pruna D: Chromosome 8p23.2-pter: a critical region for mental retardation, autism and epilepsy? Clin Genet 79: 394-395; author reply 396, 2011.

24. Cottrell CE, Bir N, Varga E, et al: Contactin 4 as an autism susceptibility locus. Autism Res 4: 189-199, 2011.

25. Cole LW, Sidis Y, Zhang C, et al: Mutations in prokineticin 2 and prokineticin receptor 2 genes in human gonadotrophin-releasing hormone deficiency: molecular genetics and clinical spectrum. J Clin Endocrinol Metab 93: 3551-3559, 2008.

26. Shaw ND, Seminara SB, Welt CK, et al: Expanding the phenotype and genotype of female GnRH deficiency. J Clin Endocrinol Metab 96: E566-E576, 2011.

27. Katsanis N, Eichers ER, Ansley SJ, et al: BBS4 is a minor contributor to Bardet-Biedl syndrome and may also participate in triallelic inheritance. Am J Hum Genet 71: 22-29, 2002.

28. Risch N, Spiker D, Lotspeich L, et al: A genomic screen of autism: evidence for a multilocus etiology. Am J Hum Genet 65: 493-507, 1999

29. Kalkman HO: A review of the evidence for the canonical Wnt pathway in autism spectrum disorders. Mol Autism 3: 10, 2012.

30. Sakai Y, Shaw CA, Dawson BC, et al: Protein interactome reveals converging molecular pathways among autism disorders. Sci Transl Med 3: 86ra49, 2011. 